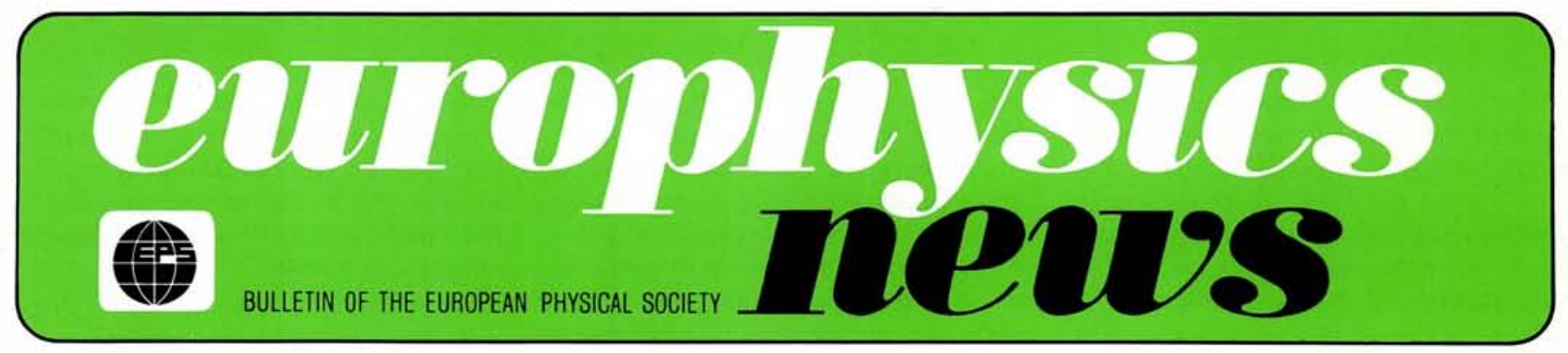

J.A.

\title{
BIOPHYSICS
}

Biophysics - a subject of great breadth and great challenge. We present here examples of different areas of research where the physicists' contribution to our understanding of living processes is of crucial importance.

\section{Role of the Physicist in Biology}

\section{E. Kellenberger, Basel}

(Dept. of Microbiology, Biozentrum of the University of Basel)

Every science progresses from a phase of description and classification or categorisation (phenomenology) into an experimental phase where causalities (natural laws) are uncovered and verified. When a sufficient number have been established they can then be connected by a comprehensive theory.

In terms of the above, physics is obviously the most advanced science; theories are predominant and most experiments are now measurements or observations designed to test predictions that theory has made. Evidently not everything in physics can be explained by means of existing theories and many phenomena still await exploration. Mostly however, these are not the "in" topics of the general consensus.

Biology is about a century behind physics and chemistry, although some biosciences like genetics were already theoretically perfect in the last century. Genetics comprises a few basic laws which are able to explain inheritance, and is comparable to classical thermodynamics. Otherwise biology in the first half of this century was still mainly descriptive, even if for practical purposes empirical "pseudo-causalities" had to be established particularly in medicine. Correlations which could be shown statistically to have a non-negligible probability of a causal origin were indispensable for establishing therapeu- tic treatments. This type of probability, devised as an expedient in the absence of a deeper understanding has to be distinguished from a natural law that contains probability as an integral part. A magnificant example of the latter is the law of independent assortment in genetics. As is well known, our chromosomes are in pairs, one stemming from each parent. The germinal cells in contrast contain only one, chosen at random from either our mother or our father. It is interesting to reflect on this randomness which accounts for the diversity of individuals and their evolution.

During the 1940s, physicists made vital contributions to the transition of biology into the experimental phase. Max Delbrück working in the group of Otto Hahn and Lise Meitner as a theoretical physicist had contacts with Timofeeff-Rossovsky from radiation biology. Learning about mutations Delbrück foresaw the possibility of linking them to the lesions produced by a physical agent acting on the atoms of the genetic material. This idea came to him at a time when the chemical nature of the carrier of genetic information was still very controversial. Nucleic acids are built up from four distinct elements, proteins from some twenty, and to the chemist it was clear that the high specificity of genes must stem from the proteins! I will not go into the very interesting story of how finally DNA became accepted as the carrier of genetic information, nor that of Watson and Crick's revolutionary new concept of the genetic code, by which all the information needed for a living organism is contained in the base sequence of this linear macromolecule.

When laying the foundations for these discoveries, Delbrück had first to introduce precise methods of experimentation and, not least, find an adequate experimental system. Luckily he chose viruses and in particular those that have bacteria as a host - the bacteriophages. Phages have been known since 1919 and a wealth of publications on them existed. Practically none, however, stood up against the criticism of this experimentalist. Delbrück had thus to start from scratch. He introduced quantitive methods and although their precision was only $\pm 20 \%$, this was sufficient to put the experimental results on a solid basis. He could determine how many phages were injected into a bacterium of a population (a culture) and at what time. He could therefore establish the

\section{Contents}

Role of the Physicist in Biology

Protein Structure

Muscle Contraction

Long-range Electrostatic Forces

NMR with Proteins and

Nucleic Acids

Microcalorimetry and

Thermodynamics

1986 Hewlett-Packard

Europhysics Prize

Computational Physics Courses 
phage life-cycle in a single cell of the culture. The new methodologies which needed only simple and inexpensive equipment and no elaborate mathematics except an ability to cope with a Poisson distribution, were rapidly taken up by many researchers and led to the identification of DNA as the carrier of genetic information, the deciphering of the code and the understanding of mutagenesis. Soon after, the mechanisms of replication and the translation of the message into proteins were explained. The abstract notions in classical genetics of Mendel were thus reduced to the structural features of a macromolecule, spawning under the general title of molecular genetics an industrial revolution and the new fields of bioengineering and biotechnology.

In parallel and in close contact with the people working on genetics, other physicists were wondering about certain basic phenomena in physiology. Haemoglobin, a protein composed of four sub-units, binds four molecules of oxygen, a process in which the first binding facilitates very strongly the binding of the next three, a phenomenon called cooperativity. A group in the Cambridge physics department headed by Bragg decided to explore this effect which it was argued must be mediated by some non-chemical and reversible change of the molecule. Perutz and Kendrew had the ingenious idea that this could be a conformational change of each protein sub-unit in the haemoglobin. They used $X$-ray diffraction as their probe and the data were analysed by Fourier transforms which needed relatively elaborate mathematics, at that time, certainly outside the reach of biologists.

The time needed for such structural investigations has been reduced by a factor of about 10 by the computer and today the methods are relatively widely employed even though very few other cases of control of physiological function through conformational change have been established by this approach. This is probably because conformationally regulated proteins are flexible and thus not easy to crystallise. More rigid proteins like enzymes are more easily prepared in crystalline form and the interaction between enzyme and protein substrate, of protein with other proteins and with nucleic acids can be studied. This has led to an enormous growth not only in our understanding of binding interactions but also of the detailed chemistry of the catalytic action of enzymes. Many physicists are involved in the structural investigations, because the needed instrumentation and methodo- logy are those of physics; even physicists still teaching physics have stimulated recent progress on proteins!

A protein should no longer be considered as consisting of a rigidly folded peptide chain, but rather of regions whose behaviour is close to that of a liquid alternating with regions that behave as a solid. Through the semi-liquid domains, small molecules are able to diffuse, e.g. in the case of haemoglobin into inside pockets. Obviously investigations into such phenomena are predestined for physicists, as are also the modern uses of NMR for medical purposes, following metabolic paths and the very new methods of investigating protein structure as described later.

The examples given above illustrate the two ways in which physicists may enter the world of biology. The one is by introducing precise experimentation together with what I call "precise physical thinking", following the example of Delbrück, the other by using complex methods involving both elaborate instrumentation and relatively complex mathematical data processing following the examples of Kendrew and Perutz.

In both cases, the problems to be solved come from biology. This is most important to realise. Physicists (and organic chemists) tend to be so convinced of the superiority of their insight, they try to explain biology in terms of their own physical theories. Many physicists have made this error. I name only one Schrödinger because he is above all suspicion of being pretentious. His "What is Life" is highly interesting to read, but applying wave mechanics to genetic coding was not useful! Later, attempts were made to apply quantum mechanics to explain and predict the carcinogenic action of chemical compounds, but these were complete failures. To me, it is obvious that insight into biology is not obtained merely by an extension of physical theories. Only "physical thinking" associated with a thorough understanding of biology is successful. This means that the physicist who wants to become a biophysicist has to make a big effort to acquire biological understanding, and not just by memorising the diversity of Nature as is still practised in many schools. There must be a deep curiosity about the wonders of life and also a certain humility. The more modern biology (including ecology and physiology) a physicist has been taught in high school, college and university, the easier it is to convert him into a biophysicist!

In the first half of my career, most of my Ph.D. students and many of my post- docs were physicists in origin. But they had to learn to ask the right questions about biological problems. This adaptation was not without pain because it is not easy for a fully fledged physicist to put aside his theoretical know-how and replace it by another.

What then is biophysics? Biology done by physicists is probably the best answer. Biophysics is not a discipline proper like genetics, biochemistry or molecular biology, but is expected to promote interdisciplinary bridging.

To teach biophysics the most successful approach is to concentrate on the physical bases of methods. They cover biophysical chemistry, the thermodynamics of macromolecules and biochemical reactions. They include all methods applicable to the investigation of structures (diffraction of X-rays and neutrons, scattering of all sorts of radiation, optical methods, ultracentrifugation, microscopies of all types, NMR, etc.) and radiobiology which has an important place in the education of modern biologists.

Biophysics should be taught to biologists and biochemists in a simple form such that they learn what help they can expect from the biophysicist. Physicists should primarily learn biology as explained above.

How are young biophysicists best formed? In my experience, the best way is for them to start with physics and chemistry but then rapidly to be introduced to modern biology. At the same time they can start to study biophysics in a more profound and extensive manner than the biologist. If they are to terminate their basic studies within a reasonable lapse of time, they cannot go as deeply into genetics, developmental biology and neurobiology as the biologist. But the goal is more rapidly achieved than by taking physicists and converting them into biophysicists after the doctorate.

To summarise and conclude, let me emphasize that those physicists who believe that biologists are not yet so evolved because of their lack of knowledge of physical theories are on the wrong track. Understanding biology is not less difficult than physics, rather the reverse! A mathematical treatment is not per se proof of a deep understanding, but a splendid tool which can be extremely helpful where it can be applied. Biophysics is a vocation demanding a feeling for biological phenomena. This is a gift which can be developed by proper education, but it is not necessarily given to every physicist. 